\title{
Magnetic fields in Planetary Nebulae: paradigms and related MHD frontiers
}

\author{
Eric G. Blackman
}

Department of Physics and Astronomy, University of Rochester, Rochester, NY 14627, USA email: blackman@pas.rochester.edu

\begin{abstract}
Many, if not all, post AGB stellar systems swiftly transition from a spherical to a powerful aspherical pre-planetary nebula (pPNE) outflow phase before waning into a PNe. The pPNe outflows require engine rotational energy and a mechanism to extract this energy into collimated outflows. Just radiation and rotation are insufficient but a symbiosis between rotation, differential rotation and large scale magnetic fields remains promising. Present observational evidence for magnetic fields in evolved stars is suggestive of dynamically important magnetic fields, but both theory and observation are rife with research opportunity. I discuss how magnetohydrodynamic outflows might arise in $\mathrm{pPNe}$ and $\mathrm{PNe}$ and distinguish different between approaches that address shaping vs. those that address both launch and shaping. Scenarios involving dynamos in single stars, binary driven dynamos, or accretion engines cannot be ruled out. One appealing paradigm involves accretion onto the primary post-AGB white dwarf core from a low mass companion whose decaying accretion supply rate owers first the pPNe and then the lower luminosity PNe. Determining observational signatures of different MHD engines is a work in progress. Accretion disk theory and large scale dynamos pose many of their own fundamental challenges, some of which I discuss in a broader context.
\end{abstract}

Keywords. Planetary Nebulae - magnetic fields - accretion - MHD - stars: AGB - stars: binaries

\section{Introduction}

Asymmetries were observed in planetary nebulae PNe before the Hubble Space Telescope but their ubiquity, the rapidity with which they develop, and collimated outflow power have led to a cultural change in the field over the past decade (e.g. Balick \& Frank 2002). Asymmetric $\mathrm{p} / \mathrm{PNe}$ have become the standard and understanding how these asymmetries arise is now fundamental rather than anecdotal. Perhaps most dramatic is that collimated momenta of the pre-PNe (pPNe) (the reflection nebulae precursor to the ionized nebulae of $\mathrm{PNe}$ ) exceed that which can be supplied by radiation pressure alone (Bujarrabal et al. 2001). This is reminiscent of the young stellar object (YSOs) subject decades ago (Reipurth \& Bally(2001)). The recent cultural change toward the view that the end states of stars (including supernovae e.g. Wang \& Wheeler 2008) are asymmetric, offers a plethora of research opportunity.

The beginning and ending of stars share the ingredients of in-fall, collapse, turbulence, and angular momentum transport. The associated increased free energy in differential rotation can be tapped to amplify fields and produce outflows, so common underlying MHD principles likely at work. Focusing on pPNe/PNe, I review the role of magnetic fields in these systems, and current evidence for their influence. I also address broader fundamental questions in magnetic field generation and angular momentum transport for which pPNe are yet another laboratory. The role of large scale fields and the need to connect different MHD engines with observations are central to the theme. 


\section{Basic properties of $\mathrm{p} / \mathrm{PNe}$}

Generally, pPNe exhibit a fast bipolar outflow embedded within a slow spherically symmetric wind from the AGB star Bujarrabal et al. 2001. Presently, data do not rule out all pPNe having gone through a strongly asymmetric outflow and all PNe having gone through an asymmetric pNE phase. Though AGB stars produce spherically symmetric outflows, the initial pPNe asymmetry emerges within $\leqslant 100 \mathrm{yr}$ (e.g. Imai et al. 2005). PNe could reflect the late stages of the mechanism that produces pPNe with less asymmetry at very late times as supersonic motions damp.

For pPNe ( Bujarrabal et al. 2001), each fast wind has a typical age $\Delta t \sim 10^{2}-10^{3} \mathrm{yr}$, speed $\sim 50 \mathrm{~km} / \mathrm{s}$, mass $M_{f} \sim 0.5 M_{\odot}$, outflow rate, $\dot{M}_{f} \sim 5 \times 10^{-4} M_{\odot} / \mathrm{yr}$, momentum $\Pi \sim 5 \times 10^{39} \mathrm{~g} . \mathrm{cm} / \mathrm{s}$, and mechanical luminosity $L_{m, f} \geqslant 8 \times 10^{35} \mathrm{erg} / \mathrm{s}$ (can be as high as $\left.10^{37} \mathrm{erg} / \mathrm{s}\right)$. The slow pPNe wind has an age $\Delta t \sim 6 \times 10^{3} \mathrm{yr}$, a speed $v_{w} \sim 20 \mathrm{~km} / \mathrm{s}$ a mass $M_{s} \sim 0.5 M_{\odot}$, outflow rate, $\dot{M}_{s} \sim 10^{-4} M_{\odot} / \mathrm{yr}$, momentum $\Pi_{s} \sim 2 \times 10^{39} \mathrm{~g} \mathrm{~cm} / \mathrm{s}$, and mechanical luminosity $L_{m, s} \sim 10^{34} \mathrm{erg} / \mathrm{s}$. For PNe, observations suggest (Balick \& Frank 2002 ) an age $\Delta t \sim 10^{4} \mathrm{yr}$ a slow wind of speed $v_{s} \sim 30 \mathrm{~km} / \mathrm{s}$ of mass $M_{s} \sim 0.1 M_{\odot}$, outflow rate, $\dot{M}_{s} \sim 10^{-5} M_{\odot} / \mathrm{yr}$, momentum $\Pi_{s} \sim 6 \times 10^{38} \mathrm{~g} \mathrm{~cm} / \mathrm{s}$, and mechanical luminosity $L_{m, s} \sim 3 \times 10^{33} \mathrm{erg} / \mathrm{s} . v$ PNe have fast winds of speed as high as $v_{f} \sim 2000 \mathrm{~km} / \mathrm{s}$, mass $M_{f} \sim 10^{-4} M_{\odot}$, outflow rate, $\dot{M}_{f} \sim 10^{-8} M_{\odot} / \mathrm{yr}$, momentum $\Pi_{f} \sim 4 \times 10^{37} \mathrm{~g} \mathrm{~cm} / \mathrm{s}$, and mechanical luminosity $L_{m, f} \sim 1.3 \times 10^{34} \mathrm{erg} / \mathrm{s}$.

The pPNe phase demands the most power and the linear momenta of fast bipolar pPNe outflows seems too large for radiation driving Bujarrabal et al. 2001. This motivates the need to tap rotational energy, either from redistribution of angular momentum within a single star or via deposition of angular rotational energy from binaries.

\section{Observations of magnetic fields}

Detection of magnetic fields in $\mathrm{p} / \mathrm{PNe}$ is not by itself a proof of their dynamical importance as the relative strength of the field and the local kinetic energies must be considered. Complementarily, a weak magnetic field at large distances from the outflow engine does not rule out a magnetically dominated launch at the engine.

Magnetic field detection is technically challenging but careful analysis has led to estimate of magnetic field strengths and geometries in several important classes of late AGB and post-AGB systems by different techniques. In water maser sources such as W43 (an evolved AGB star with a precessing jet signaturing the beginning of the pPNe stage) magnetic fields of $\sim 85 \mathrm{mG}$ have been inferred at radii of 500AU from the engine via interpretation of circular polarization of $\mathrm{H}_{2} \mathrm{O}$ masers (Vlemmings et al. 2006). The $85 \mathrm{mG}$ fields at 500AU and their inferred geometry seem consistent with what is needed to dynamically influence the flow. (See Sabin et al. 2007 for field geometry in other sources). The precession suggests a binary interaction, but whether the field is dynamo produced and/or connected to a companion within the envelope or the AGB core is uncertain. Vlemmings (2007) reviews maser field measurements of giant stars highlighting that the $\mathrm{H}_{2} \mathrm{O}$ masers probe field on $\sim 10^{2} \mathrm{AU}$ scales, $\mathrm{OH}$ masers probe $10^{3} \mathrm{AU}$ scales and $\mathrm{SiO}$ masers probe $\leqslant 10 \mathrm{AU}$. The complied measurements show that, statistically, fields fall off faster than $r^{-1}$ but slower than $r^{-3}$. This is a weak constraint on models.

From VLT spectropolarimeteric analysis of several central stars of PNe (CSPN), Jordan et al. (2005) detected magnetic field strengths of order kG. The scale of the photosphere for these system is $\sim 2 \times 10^{10} \mathrm{~cm}$. These field strengths are are consistent with the larger scale scalings presented in Vlemmings et al. (2007) and the strengths of fields needed to 
power the pPNe outflows by Poynting flux, and fields produced by dynamos in the AGB engines (Nordhaus et al. 2007).

Soker and Zoabi (2002) and Soker (2006b) have argued for weaker fields and rather than a primary driver of outflows, an indirect shaper of outflows possibly via influence on the dust distribution and geometry of radiative driven winds. But this would leave unsolved the large collimated momenta of pPNe engines Bujarrabal et al. 2001) for which radiation driving is insufficient.

\section{Dynamical magnetic shaping and/or launching}

The "launch" region of MHD outflows is the region where the magnetic force dominates the flow. This extends to a height typically no greater than $z_{c} \sim 50 R_{i}$, where $R_{i}$ is the inner-most radial scale of the engine. (e.g. the inner radius of an accretion disk). In the launch region the bulk flow is sub-Alfénic below $z_{c}$. The "propagation" region describes $z>z_{c}$ where the poloidal flow speed exceeds the Alfén speed, eventually approaching its asymptotic speed. Presently, only the propagation regions are observationally spatially resolved. Also, because there are $\sim 2$ or more orders of magnitude between the engine where dynamos and jets formation operates and the asymptotic propagation region the computational demands prohibit simulatating the combined physics of the launch region, jet formation and asymptotic propagation. As emphasized in sec 7., even the nonlinear physics of just an accretion disk has been prohibitively computationally expensive. We must patch together different pieces of physics from different approaches and scales to extract a complete picture.

\subsection{Shaping with imposed fields}

In a semi-analytic approach, Chevalier and Luo (1994), imposed a magnetic field geometry such that the toroidal field falls off more slowly than the poloidal field and at large distances and a spherical wind shocks the ambient gas increasing the toroidal field via compression. For their parameters, the magnetic shaping occurs only after the shock.

García-Segura (1997) and García-Segura et al. (1999) were the first to simulate the direct effect of imposed toroidal fields on PNe shaping by driving a hydrodynamic wind into a pre-magnetized medium. The field strengths were consistent with those of sec. 3 above. and their influence (along with stellar rotation, and photoionization) on the shaping was summarized in simple schematic (García-Segura 1999). Rotation + magnetic fields leads to strong collimation compared to the weaker collimation of just the rotational influence on radiatively driven winds. Gardiner \& Frank (2001) discussed that the shaping observed in such simulations were outside the restrictive parameter regime of Chevalier \& Luo (1994) and that the influence of reasonable strength fields should generically affect the outflow before the shock compression. .

\subsection{Launching and shaping with imposed initial fields}

Because the observations require both a mechanism of launch and shaping, it is necessary to consider the role of magnetic fields in both. García-Segura et al. (2005) imposed a toroidal field the surface of the AGB star to launch an outflow via the field pressure gradient and studied the propagation and asymptotic collimation over 2 orders of magnitude in scale. Given field strengths of $\sim 40 G$ (consistent with observations) at the surface of the AGB star, outflows consistent with the required power and shaping were observed.

Matt et al. (2006) added additional physics in demonstrating how an initially weak field can grow linearly in time by extracting rotational energy of its anchoring base, and produce a rapid bipolar outflow from the gradient in toroidal field pressure. They took 
a gravitating spherical core surrounded by an initially hydrostatic envelope of ionized gas. A dipolar magnetic field was anchored on the core, threading the envelope. The core was set to rotate initially at $10 \%$ of the escape speed. The toroidal field amplified from the differential rotation between core and envelope and when the toroidal field pressure gradient overcame the envelope binding energy, envelope material was rapidly expelled in a quadrupolar outflow: The wound up diploe field had maximum toroidal field at intermediate poloidal angles in each hemisphere, so material was both along the poles also squeezed out from the equator.

\subsection{Launching via dynamo engines}

The models of the previous two subsections do not fully address where the dynamically important large scale fields come from. Even the Matt et al. (2006) paper invoked linear, laminar field growth and treating the rotator base as a boundary condition.

Papers such as those by Pascoli (1993,1997) Blackman et al. (2001a,b), Nordhaus et al. (2007) use flavors of a mean field dynamo theory for stellar or disk engines to estimate field strengths and Poynting fluxes that could arise. These papers are not simulations and do not track global field geometry, or follow the production and evolution of outflows from the field production region. Furthermore, the nonlinear physics of dynamos (see sec. 7) require approximations when emedding them in astrophysical scenarios. Despite their shortcomings, such toy models do produce promising results with respect to field strengths and Poynting fluxes

\section{Single star vs. binary models}

\subsection{Single star models not yet ruled out}

Binaries can easily supply the needed free energy to amplify fields via differential rotation (ordhaus \& Blackman 2006). Isolated star MHD models cannot be ruled out, but there are caveats (Soker 2006b; Nordhaus et al. 2007) as I now discuss.

Blackman et al. (2001) investigated an isolated interface dynamo model operating at the base of the AGB convective zone in which angular momentum is conserved on spherical shells as the star evolves off the main sequence and the resulting rotation profile provides the available differential rotational energy from which the field is amplified. To drive bipolar $\mathrm{pPN} / \mathrm{PN}$, the corresponding dynamo must operate through the lifetime of the AGB phase $\left(10^{5} \mathrm{yr}\right)$ until radiation pressure has bled most of the envelope material away. Only then can the Poynting flux unbind the remaining material. But the dynamo would drain differential rotation on time-scales short compared to the AGB lifetime. Only if differential rotation is extracted from deep within the core or re-seeded by convection can the drain be overcome (Nordhaus et al. 2007). Viability of the single star dynamo outflow model, depends on whether or not the differential rotation can be re-supplied over the AGB lifetime via convection as in the Sun.

\subsection{Common envelope binary scenarios}

In a common envelope (CE) Iben \& Livio (1993) the companion drags on the envelope of the primary, transferring angular momentum and energy. If the envelope cooling time is long enough, a fraction $\alpha \geqslant 0.1$ of the loss in gravitational energy can spin up and unbind the envelope. Reyes-Ruiz \& Lopez (1999) discuss the secondaries for which accretion disks will form around the primary core. Brown dwarf (BD) $\left(0.003 M_{\odot}<M_{\text {crit }} \sim 0.07 M_{\odot}\right)$ radii increase with decreasing mass while their Roche radii decrease with decreasing mass. Such objects unstably lose mass. Since the circularization radius lies outside of the primary's core, a disk can form within a few orbits. This contrasts the $M>M_{\text {crit }}$ case 
for which the stellar radius decreases with decreasing mass more strongly than the Roche radius. Supercritical companions have a circularization radius within the primary's core. Material leaving the secondary would then initially spiral swiftly into the primary rather than orbit quasi-stably. Nordhaus \& Blackman (2006) show that accretion from planets and low mass stars may also be important (and perhaps more common because of the brown dwarf desert Grether \& Lineweaver(2006)) and can supply the needed accretion power for pPNe. Planets are of sub-critical mass and a disk could form as per a BD, before unbinding the envelope.

A companion star is supercritical so if stellar incurs Roche lobe overflow after CE, sustained accretion requires the binary to lose angular momentum. This could happen as the overflowing secondary drags on residual inner envelope material. Even though the circularization radius for $M_{2}>M_{\text {crit }}$ is inside the core, the in-spiraling material still incurs differential rotation and could amplify magnetic fields. A significant energy release via accretion in the $M_{2}>M_{\text {crit }}$ case could occur on impact to the inner core, producing dwarf novae bursts masked by the stellar envelope. If this initial accretion phases can drop $M_{2}$ below $M_{c r i t}$, keep $M_{2}$ filling its Roche lobe, and leave enough angular momentum to form a Keplerian disk, then accretion could proceed as for the initial $M_{2}<M_{\text {crit }}$ case.

Huggins (2007) suggests a $\sim O(100)$ year delay between ejection of cicumbinary dust tori and jets in pPNe. If a fraction of ejected envelope material becomes the dust torus, the delay could be the time for the companion to lose enough mass to move the circularization radius outside the core for the supercritical case, or a viscous time.

\section{Accretion disk outflows in pPNe and PNe}

Accretion disk outflows have a mechanical power Blackman et al. (2001b)

$$
L_{m} \sim \frac{G M_{*} \dot{M}_{a} \epsilon}{2 R_{i}}=4.5 \times 10^{36} \epsilon_{-1} \frac{M_{*} \dot{M}_{-4}}{R_{i, 10}},
$$

where $\epsilon$ is the efficiency of conversion from accretion to outflow, $R_{i}$ is the inner disk radius, $\dot{M}_{a}$ is the accretion rate, $G$ is Newton's constant, $M_{*}$ is the central stellar mass, $R_{i, 10} \equiv R_{i} / 10^{10} \mathrm{~cm}, \epsilon_{-1} \equiv \epsilon / 0.1$ and $\dot{M}_{a,-4}=\dot{M}_{a} / 10^{-4} M_{\odot} /$ yr. For an MHD outflow, Eq. (6.1) equals the Poynting flux at the launch surface. Propagation into a low density medium produces an asymptotic outflow speed $\sim \Omega r_{A}$ where $\Omega$ is the angular speed of field anchor point and $r_{A}$ is the radius where the poloidal outflow speed equals the Alfvén speed. This product is typically a few times the escape speed of the inner most radius of a disk and is thus at least $v_{\text {out }} \sim v_{\text {esc }}=1600\left(\frac{M_{*}}{R_{*, 10}}\right)^{1 / 2} \mathrm{~km} / \mathrm{s}$

\subsection{Accretion onto primary}

The $v_{\text {out }}$ above depends only weakly on $\dot{M}_{a}$ via $R_{i}$, but strongly on the inertia of material blocking the outflow: Momentum conservation gives

$$
v_{\text {obs }}=\frac{M_{f} v_{\text {out }}}{f_{\Omega} M_{\text {env }}+M_{f}} \sim 80 \mathrm{~km} / \mathrm{s},
$$

where $M_{f} / M_{\odot}=3.3 \times 10^{-4} \epsilon_{-1} \dot{M}_{a 0,-3} \int_{1}^{1000} \tau^{-5 / 4} d \tau$ is the mass in one of the fast collimated outflows, $M_{\text {env }}$ is the envelope mass, $f_{\Omega} \sim 0.2$ is the solid angle fraction intercepted by the collimated outflow, and $\tau \equiv t / 1 \mathrm{yr}$ is used to incorporate $\dot{M}_{a} \propto t^{-5 / 4}$ of Reyes-Ruiz \& Lopez (1999). The numbers are scaled to pPNe so $M_{e n v}>>M_{f}$ and for an envelope of mass $2 M_{\odot}$, the intercepted mass is $0.2 M_{\odot}$ for $f_{\Omega}=0.2$. 
Eq. (6.2) is the observed speed of the fast when blocked and loaded by the envelope. By the end of the pPNe phase, the envelope is quite extended, reducing the optical depth and revealing material moving at the "free streaming" fast wind speed. Assuming a dust-togas mass ratio of $1 / 100$ and micron sized grains of density of $2 \mathrm{~g} / \mathrm{cm}^{3}$, the optical depth from dust is $\tau_{d} \sim 2.5 \times 10^{-3}\left(\frac{n_{d}}{2.5 \times 10^{-13} \mathrm{~cm}^{-3}}\right)\left(\frac{\sigma_{d}}{10^{-8} \mathrm{~cm}^{2}}\right)\left(\frac{R}{10^{18} \mathrm{~cm}}\right)$, scaled for PNe. For pPNe, the density increases by $\geqslant 10^{4}$ and $R$ is down by a factor of 10 , so $\tau_{d} \geqslant 2.5$. The different optical depths of pPNe and PNe can thus explain why observed PNe fast winds can have $v_{f}=v_{\text {out }}>1600 \mathrm{~km} / \mathrm{s}$, whilst those of $\mathrm{pPNe}$ have $v_{f}=v_{\text {out }}<100 \mathrm{~km} / \mathrm{s}$.

Time dependent accretion outflows described with Eqs. (6.1) and (6.2) are consistent with the high pPNe outflow mechanical luminosity and the fast PNe wind speed of Sec. 1 when $M_{*} / R_{*}$ corresponds to a WD. Reyes-Ruiz \& Lopez (1999) considers a companion of mass $M_{2} \sim 0.03 M_{\odot}<M_{\text {crit }}$ and a Shakura-Sunyaev (Shakura \& Syunyaev(1973)) viscosity parameter $\alpha_{s s} \sim 0.01$, for which the accretion rate then decays as $\dot{M}_{a} \sim 1.6 \times$ $10^{-3} t^{-5 / 4} M_{\odot} /$ yr. Using this in (6.1) with $\epsilon=0.1$ for $t=100 \mathrm{yr}$ with $R_{i}=2 \times 10^{9} \mathrm{~cm}$ and $M_{1}=0.6 M_{\odot}$ gives $L_{m, f} \sim 4.3 \times 10^{39}(t / 1 \mathrm{yr})^{-5 / 4}$. This provides the needed power demands of Sec. 1 for pPNe after $1000 \mathrm{yr}$ and for PNe after $10^{4} \mathrm{yr}$.

A more careful analysis of the predicted jet speed evolution is needed for specific outflow models as the envelope evolves in order to test the idea contained within the rough estimates just discussed. See also Garicia-Diaz et al. (2008) for a non-accretion based time dependent comparison of jet outflow speed from simulations with observations.

\subsection{Accretion onto secondary}

It is also possible for accretion disks to form around the secondary (Soker \& Livio 1994; Mastrodemos \& Morris 1998; Soker 2005). The Bondi wind accretion rate is $\frac{\dot{M}}{\dot{M}_{s}}=\left(\frac{M_{2}}{M_{1}}\right)^{2} \frac{\left(v / v_{s}\right)^{4}}{\left[1+\left(v / v_{s}\right)^{2}\right]^{3 / 2}}$, where $v$ is the orbital speed of the secondary and $v_{w}$ is the slow wind speed from the primary. In general, for $M_{2}<M_{1}$, reasonable parameters provide an accretion rate compatible $\mathrm{PNe}$ luminosities if the companion is either main sequence or compact star, but the ubiquity of high collimated fast wind pPNe powers (Bujarrabal et al. 2001) would require an overabundance of accreting WD companions.

\section{Key issues in large scale MHD dynamo and accretion theory}

\subsection{Large scale dynamo theory: recent developments, open questions}

Large scale dynamo (LSD) theory describes the sustenance of magnetic fields on time or spatial scales larger than turbulent scales. Whether large scale fields for jets are advected or LSD produced has been debated but but ultimately, the equations that include a the competition between turbulent transport, flux accretion, as well as LSD action need to be solved. Field reversals in the sun prove that an LSD can and must operate therein.

For $\sim 50$ years, a problem with textbook LSD theory (e.g. Moffatt (1978)) has been the absence of a proper saturation theory that predicts how strong the large scale fields get before non-linearly quenching via the backreaction of the field on the driving flow kicks in. But substantial progress toward a nonlinear mean field theory has emerged in the last decade via a symbiosis between analytical and numerical work. Coupling the dynamical evolution of magnetic helicity into the dynamo equations turns out to be fundamental for predicting the saturation seen in simulations. For recent extensive reviews see (Brandenburg \& Subramanian 2005; Blackman 2007).

Much of the work in LSD theory, has focused on systems that are initially globally reflection asymmetric (GRA). This means a global pseudoscalar is imposed by the 
boundary conditions-for example, rotation and stratification lead to the kinetic helicity pseudoscalar, common to the standard textbook (Moffatt (1978)) " $\alpha_{d y n}$ effect" of mean field dynamos. However magnetic helicity is actually the unifying quantity for LSD. There are two classes of GRA LSD for which an electromotive force aligned with the mean magnetic field is essential (see e.g. Blackman 2007). The first is flow driven helical dynamos (FDHD) which occur inside of astrophysical rotators. Here the initial energy is dominated by flows and the field responds. These are linked to a corona by magnetic buoyancy. In coronae, the second type of LSD, the magnetically driven helical dynamo (MDHD) can operate. This characterizes relaxation of magnetic structures to larger (jet mediating) scales in a magnetically dominated environment subject to the injection of smaller scale magnetic helicity. The MDHD is directly analogous to laboratory plasma dynamos that occur in reverse field pinches (RFPs) and Spheromaks.

LSDs always involve some helical growth of the large scale field which is coupled to a helical scale fields of opposite sign. When small scale magnetic or currently helicity evolution is coupled to the large scale field growth, the modern mean field 'dynamo $\alpha_{d y n}$ effect that predicts the correct saturation becomes the difference between kinetic helicity and current helicity: For a FDHD simulated in a closed box (Brandenburg 2001), the current helicity builds up as the large scale field grows and quenches the FDHD (Blackman \& Field 2002). Complementarily, for an MDHD, the system is first dominated by the current helicity and a growing kinetic helicity then acts as the backreaction (Blackman \& Field 2004). Both FDHD and MDHD are accessible with in the same framework, all unified by tracking magnetic helicity evolution, and aided by thinking of the field as ribbons rather than lines (Blackman \& Brandenburg 2003). More work on how the fields evolve from within the rotator to produce the global scale fields in coronae which in turn produce jets are needed as most work on dynamo theory has focused on the FDHD.

Because the buildup of small scale magnetic (or current) helicity quenches the LSD, preferential ejection of small scale helicity vs. large scale helicity through a boundary can in principle alleviate this quenching (Blackman \& Field 2000; Vishniac Cho 2001; Sur et al. 2007). Numerical simulations support this general notion, particularly when shear is present and when surfaces of shear align toward open boundaries (Brandenburg \& Sandin 2004; Kapyla et al. 2008), thereby allowing needed helicity fluxes.

LSD action has also been observed in non GRA simulations (e.g. Yousef et al. 2008; Lesur \& Ogilvie 2008) implying that the minimum global ingredients for this class of analytic LSD is shear, plus turbulence that feeds azimuthal field back to toroidal field. There is work in progress to understand the simulations guided by analytic models (e.g. Vishniac \& Brandenburg 1997; Blackman 1998; Kleeorin \& Rogachevskii 2003; Schekochihin et al. 2008). The non GRA LSDs grow large scale fields on scales larger than the turbulence but smaller than the global scale. In coherence regions, there is a field aligned electromotive force (EMF), and thus an intermediate scale source of magnetic helicity that may switch signs between coherence regions and globally average to zero. It may be that the non-GRA LSD action always involves a local helicity flux between coherence regions.

\subsection{Accretion disks: more questions and the need for large scale fields}

The magneto-rotational instability (MRI) has emerged as a leading candidate for angular momentum transport in accretion disks (e.g. Balbus \& Hawley 1998) Although the MRI exists without the LSD, they are likely coupled in nature. I explain this below.

There is a disconnect between what shearing box simulations have told us about the MRI vs. how the instability might operate in nature. To date, simulations have primarily told us that the MRI is plausible but do not produce a robust theory of saturation or 
robust values of transport coefficients for modelers. This may frustrate, but patience (for several more decades) is required, as the computational and conceptual demands are substantial. For example, to achieve better resolution, most first generation MRI simulations (except Brandenburg et al.1995) did not use explicit viscosity or magnetic diffusivity (see discussion in e.g. Fromang et al. 2007). The magnetic Prandtl number affects the magnetic spectrum and the transport coefficients. In addition, the value of the angular momentum transport coefficient $\alpha_{s s}$ depends strongly on the box size and the strength of the initially imposed weak mean field strength (Pessah et al. 2007). Interestingly, $\alpha_{s s}$ varies $\sim 4$ orders between simulations, but $\alpha_{s s} \beta$, where $\beta$ is the ratio of thermal to magnetic pressure, is nearly a constant (Blackman et al. 2007).

In addition to the need for explicit diffusivities, perhaps the most important frontier is actually role of large scale magnetic fields in angular momentum transport and thus, non-local contributions to magnetic stresses that transport angular momentum. Its importance is motivated from three different paths:

(1) Large scale fields are evidenced from theory, simulation, and observations of coronae and jets. Large field structures more easily survive the buoyant rise to coronae without being shredded by turbulence within the disk. Plausibly, the integrated stress for structures on scales above that which survives the buoyant rise would contribute to the large scale magnetic stress. But shearing boxes that study only a local region non-local large scale magnetic stress is excluded. In a real system this could be the most important part.

(2) Shearing box simulations artificially impose a steady-state because differential rotation is imposed as a steady forcing not subject to the backreaction of the amplified field. Hubbard and Blackman (2008b), argue that this may be more restrictive that previously recognized: In a real disk, energy in differential rotation is susatined only by accretion itself. If a steady-state is to be maintained via turbulent transport alone then there must be $100 \%$ power throughput from differential rotation to the turbulent cascade. This is not guaranteed if large scale fields drain power. An accessible steady-state solution must then incorporate stresses from large-scale fields in addition to turbulent transport.

(3) The role of large scale magnetic fields is consistent with the implications of (Pessah et al. 2007) which shows that MRI stresses in simulation boxes where the radial extent is of order the vertical scale height scale with the ratio of box size to scale height. The contribution of large scale fields would increase as the radial and vertical scales are increased, highlighting a strongly non-local contribution to stresses that transport angular momentum. Evidence for non-local MRI behavior is also seen in Bodo et al. (2008). Box sizes for thin disks must be extended in the radial direction and accordingly in the vertical direction as buoyant loops tend to have radial scales comparable to vertical scales.

Ultimately, mean field accretion disk theory should be coupled to an LSD theory in a real disk as they are actually artificially separated components of what should be a single mean field theory. Note that for any turbulent disk, any assumption of axisymmetry for bulk quantities automatically requires that theory to be a mean field theory. This is often veiled by a mere replacement of the actual viscosity with a turbulent viscosity as in the Shakura-Sunyaev approach (Shakura \& Syunyaev(1973)). Hubbard \& Blackman (2008a) for example, suggest that in a formal mean field theory, a term involving fluctuations of density and velocity might be interpreted as an additional transport coefficient in the mean surface density equation, restricting available steady states. Balbus et al. (1994)incorporated this term into a redefinition of accretion rate. 


\section{Much work needed to link theory and observation}

It remains a major challenge to rigorously couple the engine physics of field generation and accretion to jet launch and jet propagation in a unified theory or simulation that make distinct observational predictions for specific engines. This enterprise spans several subfields of theoretical astrophysics, let alone the specific application to $\mathrm{p} / \mathrm{PNe}$.

Here however, is a list of possible lower hanging fruit for linking theory with observations: (1) Evaluate the kinematic constraints/predictions of outflows from the scenario of accretion onto the primary and compare the distribution of inferred fast outflow speeds to what would be expected from known binary statistics of low mass stars. This would help constrain the commonality of accretion onto the primary vs. secondary as the latter has a broader range of masses. (2) The more massive the companion, the more CE models would predict mostly Oxygen rich rather than Carbon rich post AGB systems because the binding energy for the early AGB phases is higher. Low mass companions like planets may terminate the AGB only in the thermal pulse phase. (3) Crystalline dust in postAGB systems can be produced if a binary induced spiral shock anneals silicates (Edgar et al.(2008)). Is this universal? (4) CE evolution would predict equatorial outflows that precedes any accretion driven poloidal jet. Is this consistent with the delay of Huggins (2007) and the geometry of equatorial outflows? (6) Are fast outflows contaminated by material that could represent accretion disk residue of shredded low mass companions? (7) Are time scales of observed outflow precession consistent with the gravitational influence of a binary? (8) Can double peaked line profiles be detected to identify accretion disks within the launch region? (9) Can shrouded novae outbursts from a $M_{2}>M_{\text {crit }}$ companion feeding the primary be detected in X-rays? (10) Improved statistics on the fraction of bipolar pPNe, the fraction of suitable precursor binaries for CE, and the fraction of stars which evolve to be pPNe will improve evaluation as to whether all $\mathrm{PNe}$ incur asymmetric phase. (12) Can the approach of Ferreira et al. (2006) be generalized to pPNe outflows?

\section{References}

Balbus, S. A. \& Hawley, J. F. 1998, Reviews of Modern Physics 70, 1

Balbus, S. A., Gammie, C. F. \& Hawley, J. F. 1994, MNRAS 271, 197

Balick, B. \& Frank, A. 2002, ARAA 40, 439

Blackman, E. G. 1998, ApJ 496, L17

Blackman, E. G., \& Field, G. B. 2000, MNRAS 318, 724

Blackman, E. G., Frank, A., Markiel, J. A., Thomas, J. H., \& Van Horn, H. M. 2001a, Nature 409, 485

Blackman, E. G., Frank, A., \& Welch, C. 2001b, ApJ 546, 288

Blackman, E. G. \& Field, G. B. 2002, Phys. Rev. Letters 89, 265007

Blackman, E. G. \& Brandenburg, A. 2003, ApJ 584, L99

Blackman, E. G. 2007, New Journal of Physics 9, 309

Blackman, E. G., Penna, R. F. \& Varnière, P. 2008, New Astronomy 13, 244

Bodo, G., Mignone, A., Cattaneo, F., Rossi, P., \& Ferrari, A. 2008, A\&A 487, 1

Brandenburg, A., Nordlund, A., Stein, R. F., \& Torkelsson, U. 1995, ApJ 446, 741

Brandenburg, A. 2001, ApJ 550, 824

Brandenburg, A. \& Sandin, C. 2004, A\& A 427, 13

Brandenburg, A. \& Subramanian, K. 2005, Phys. Rep. 417, 1

Bujarrabal, V., Castro-Carrizo, A., Alcolea, J., \& Sánchez Contreras, C. 2001, A $\& A$ 377, 868

Chevalier, R. A. \& Luo, D. 1994, ApJ 421, 225

Edgar, R. G., Nordhaus, J., Blackman, E. G., \& Frank, A. 2008, ApJ 675, L101

Ferreira, J., Dougados, C., \& Cabrit, S. 2006, A\& A 453, 785 
Fromang, S., Papaloizou, J., Lesur, G., \& Heinemann, T. 2007, A\&A 476, 1123

García-Segura, ,G., 1997, ApJ 489, L189

García-Díaz, M. T., López, J. A., García-Segura, G., Richer, M. G., \& Steffen, W. 2008, ApJ 676,402

García-Segura, G., Langer, N., Różyczka, M., \& Franco, J. 1999, ApJ 517, 767

García-Segura, G., López, J. A., \& Franco, J. 2005, ApJ 618, 919

Gardiner, T. A. \& Frank, A. 2001, ApJ 557, 250

Grether, D. \& Lineweaver, C. H. 2006, ApJ 640, 1051

Herpin, F., Baudry, A., Thum, C., Morris, D., \& Wiesemeyer, H. 2006, A\&̋A 450, 667

Hubbard, A., \& Blackman, E. G. 2008a, MNRAS 390, 331

Hubbard, A. \& Blackman, E. G. 2008b, MNRAS , submitted

Huggins, P. J. 2007, ApJ 663, 342

Iben, I. J. \& Livio, M. 1993, PASP 105, 1373

Imai, H., Nakashima, J.-I., Diamond, P. J., Miyazaki, A., \& Deguchi, S. 2005, ApJ 622, L125

Jordan, S., Werner, K., \& O'Toole, S. J. 2005, A\&A 432, 273

Käpylä, P. J., Korpi, M. J., \& Brandenburg, A. 2008, A\&A 491, 353

Lesur, G. \& Ogilvie, G. I. 2008, A\&A 488, 451

Matt,S., Frank A. \& Blackman, E.G., 2006, ApJ 647, L45

Mastrodemos, N. \& Morris M., 1998, ApJ 497, 303

Moffatt, H. K. 1978, Magnetic field generation in electrically conducting fluids, Cambridge University Press, p.353

Nordhaus, J. \& Blackman, E. G., 2006, MNRAS 370, 2004

Nordhaus,J., Blackman, E. G. \& Frank, A., 2007, MNRAS 376, 599

Pascoli, G. 1993, Journal of Astrophys. and Astr. 14, 65

Pascoli, G. 1997, ApJ 489, 946

Pessah, M. E., Chan, C.-K., \& Psaltis, D. 2007, ApJ 668, L51

Pudritz, R. E., 2004, Les Houches Summer School, 78, 187

Reipurth, B. \& Bally, J. 2001, ARAA 39, 403

Reyes-Ruiz, M. \& Lopez, J. A. 1999 ApJ 524, 952

Rogachevskii, I. \& Kleeorin, N. 2003, Phys. Rev. E 68, 036301

Sabin, L., Zijlstra, A. A., \& Greaves, J. S. 2007, MNRAS 376, 378

Schekochihin, A. A., et al., J. C., Rogachevskii, I., \& Yousef, T. A. 2008, arXiv:0810.2225

Shakura, N. I. \& Syunyaev, R. A. 1973, A\&A 24, 337

Steffen, W., García-Segura, G., \& Koning, N. 2008, arXiv:0809.5263

Soker,N., 2005, $A J$ 129, 947

Soker,N, 2006a, ApJ 645, L57

Soker, N. 2006b, PASP 118, 260

Soker, N., \& Livio, M. 1994, ApJ 421, 219

Soker, N. \& Zoabi, E. 2002, MNRAS 329, 204

Sur, S., Shukurov, A., \& Subramanian, K. 2007, MNRAS 377, 874

Tout, C. A. \& Pringle, J. E. 1992, MNRAS 256, 269

Vishniac, E. T. \& Brandenburg, A. 1997, ApJ 475, 263

Vishniac, E. T. \& Cho, J. 2001, ApJ 550, 752

Vlemmings, W. H. T. 2007, in Astrophysical Masers and their Environments, IAU Symposium 242, p. 37

Vlemmings, W. H. T., Diamond, P. J., \& Imai, H. 2006, Nature 440, 58

Vlemmings, W. H. T., \& van Langevelde, H. J. 2008, A\&A 488, 619

Wang, L. \& Wheeler, J. C. 2008, ARAA 46, 433

Yousef, T. A. et al., 2008, Phys Rev. Lett. 100, 184501

\section{Discussion}

De Gouveia dal Pino: You have mentioned a sort of magneto-centrifugal process out of a magnetized accretion disk as a possible driving mechanism for PNe winds, or in other words, the same mechanism proposed for jets. But this mechanism predicts a 
very collimated bipolar outflow and in general PNe winds do not have a very collimated morphology. So how do you explain this in terms of the process above? Is it due to a combination with the outer envelope?

BLACKMAN: I should emphasize that the main reason for considering MHD bipolar outflow models is because the observations of all pre-planetary nebulae and many planetary nebulae do in fact have a strongly collimated or bipolar component as revealed by observations in the past decade. That being said, in all of these objects one also always has the radiation driven stellar wind in addition to whatever MHD outflow might be present. The radiation driven wind will always provide a quasi-spherical or less strongly collimated component to the morphology.

RÜDIGER: You suggested that differential rotation may be reseeded by convection. However, in accretion disks the rotation vector $\vec{\Omega}$ is parallel to the density gradient associated with the convectively instable direction whereas I think one would need the rotation vector perpendicular to this for such a mechanism. Can you address this?

BlaCKMAN: Yes. The convective reseeding of differential rotation that I was speaking of was in the context of an isolated star not for a thin disk. The point was that an AGB star would need a mechanism to reseed the differential rotation to maintain a magnetic field long enough to power a strong bipolar pPNe outflow in the absence of a binary companion. For a star there is a significant $\vec{\Omega} \perp \nabla \rho$. The presence of an accretion disk from a binary would circumvent this need for reseeding if the jet came from the disk.

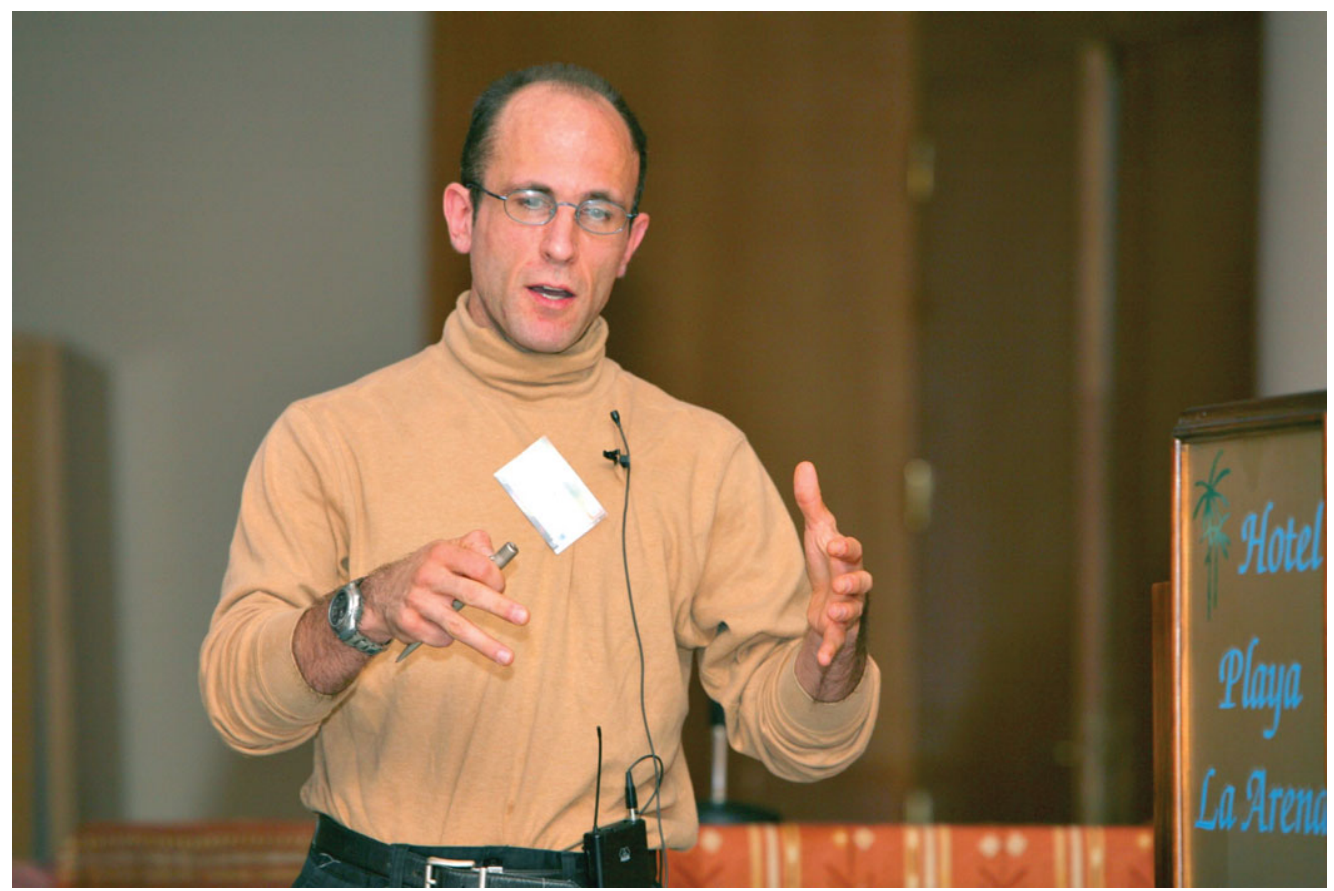

Eric Blackman 


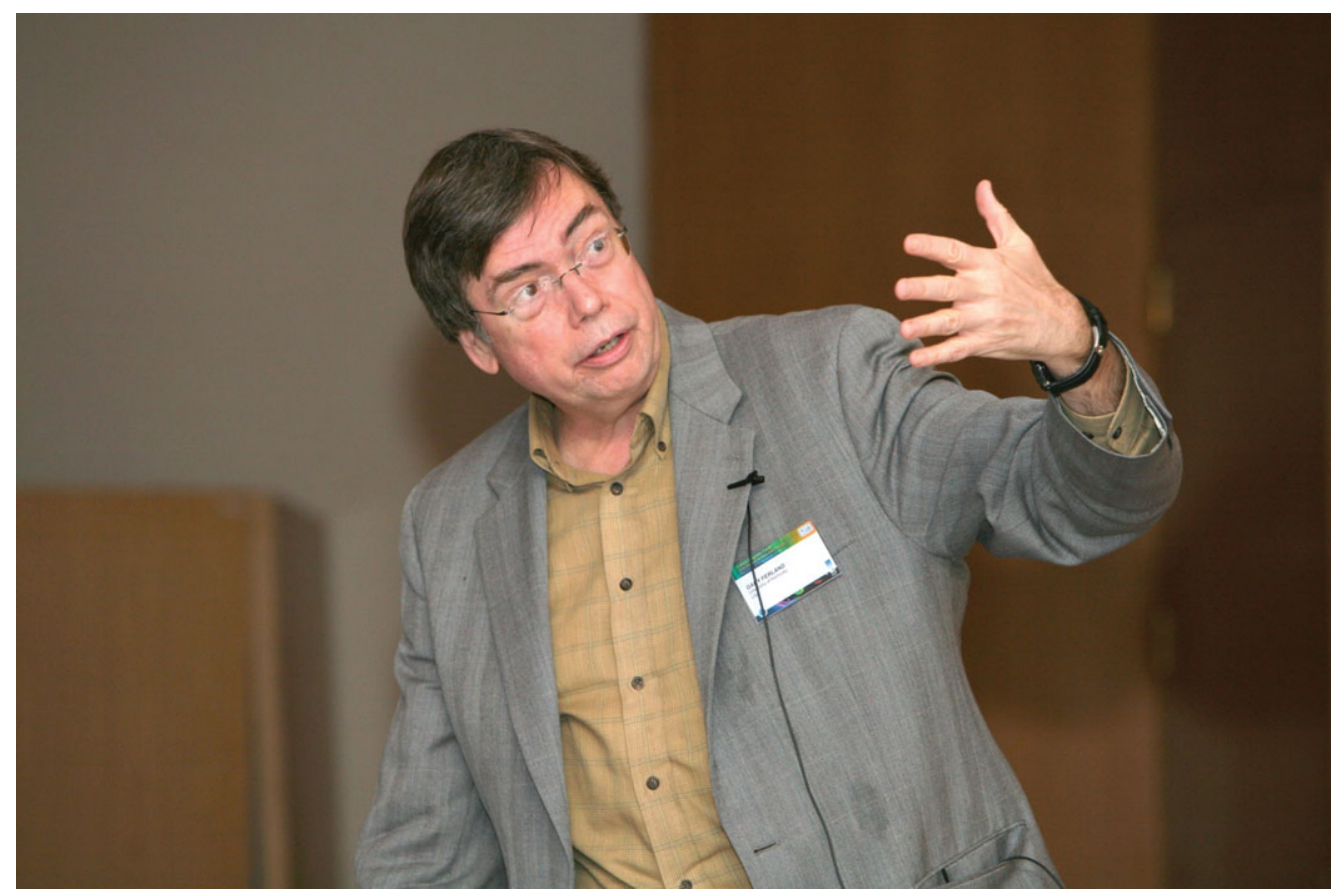

Gary Ferland

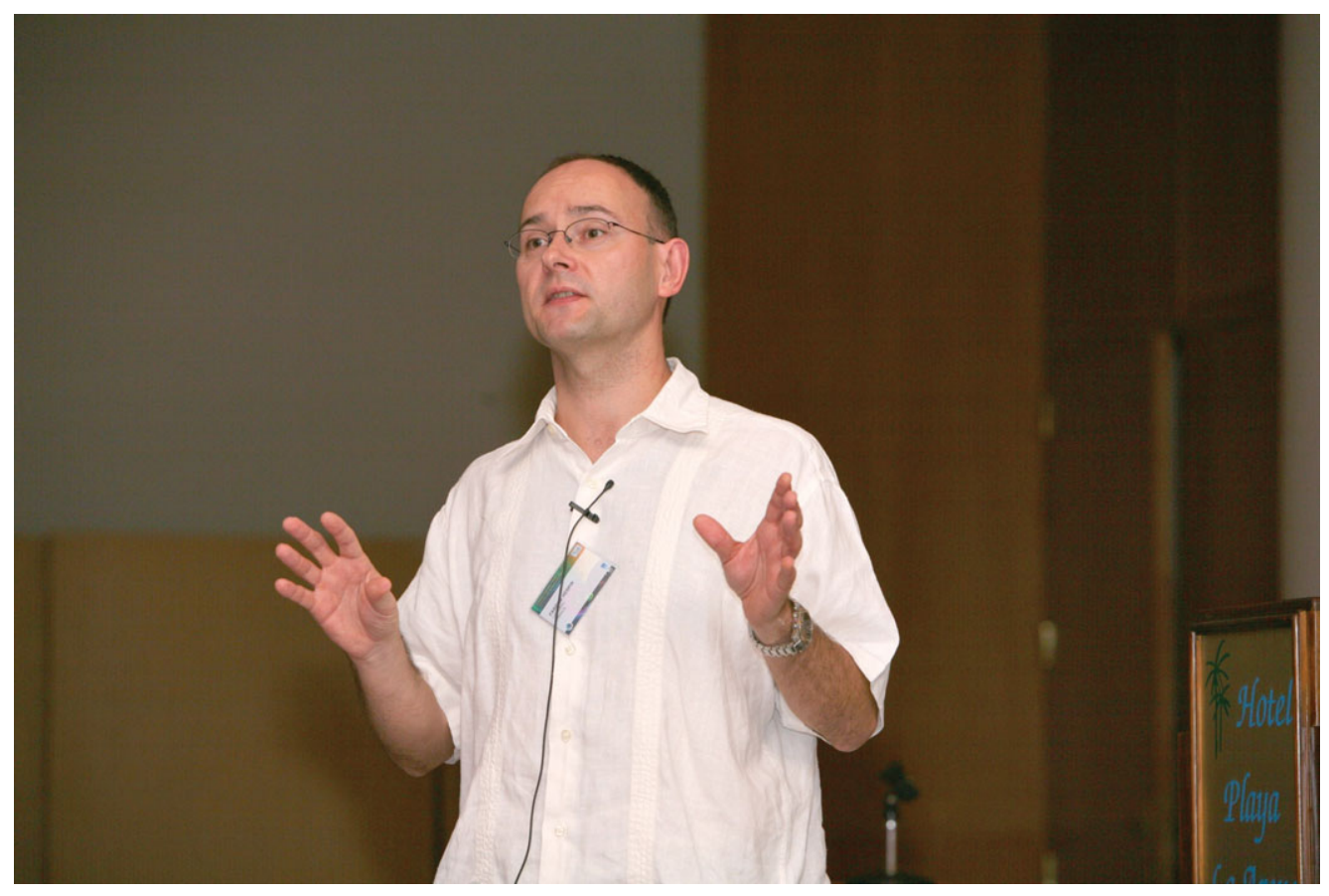

Fabrice Herpin 